\title{
Apendagite Epiplóica: Tratamento Conservador
}

\author{
Epiploic Appendagitis: Conservative Treatment
}

\author{
GUSTAVO PIGNATON ${ }^{1}$, ADRIANADE ALMEIDABORGES $^{2}$, RENATO MENDONÇA $^{2}$, \\ CLÁUDIA RIBEIRO², MARIA CHIARA CHINDAMO ${ }^{3}$
}

${ }^{1}$ Residência Médica em Gastroenterologia pela UFRJ. Especialização em Endoscopia Digestiva pelo INCA; ${ }^{2}$ Residência Médica em Gastroenterologia pela UFRJ; ${ }^{3}$ Professora da Faculdade de Medicina da UFRJ - Rio de Janeiro - RJ - Brasil.

PIGNATON G; BORGES AA; MENDONÇA R; RIBEIRO C; CHINDAMO MC. Apendagite Epiplóica: Tratamento Conservador. Rev bras Coloproct, 2008;28(3): 350-352.

Resumo: Apendagite epiplóica (AE) é uma doença inflamatória abdominal incomum, de bom prognóstico, que vem sendo mais freqüentemente diagnosticada em virtude dos avanços nos métodos de imagem. $O$ achado clínico mais freqüente é dor em quadrante inferior esquerdo. O diagnóstico é obtido por meio da tomografia computadorizada. A recuperação do quadro é completa sob tratamento conservador.

Descritores: Dor Abdominal; Disgnóstico; Apendagite Epiplóica; Tratamento.

\section{INTRODUÇÃO}

A apendagite epiplóica (AE) é uma condição clínica incomum, benigna e autolimitada. Resulta da torção ou trombose venosa espontânea das veias que drenam os apêndices epiplóicos. Manifesta-se por dor abdominal aguda, localizada principalmente em quadrante inferior esquerdo (QIE). O diagnóstico se faz por tomografia computadorizada (TC) de abdome. $\mathrm{O}$ tratamento é conservador.

\section{RELATO DE CASO}

Paciente do sexo masculino, 31 anos, iniciou quadro de dor em quadrante inferior esquerdo do abdome há 3 dias. Realizado atendimento médico no Hospital Barra D'Or, foi submetido a exames complementares. Exames laboratoriais incluindo hemograma e EAS foram normais. Realizou-se TC de abdome que evidenciou imagem ovalar medindo $3,2 \mathrm{~cm}$, com densidade de gordura e centro radioluscente. (Figuras 1 e 2) Feito diagnóstico de apendagite epiplóica. Prescritos analgésicos e antiinflamatórios para tratamento ambulatorial com evolução favorável.

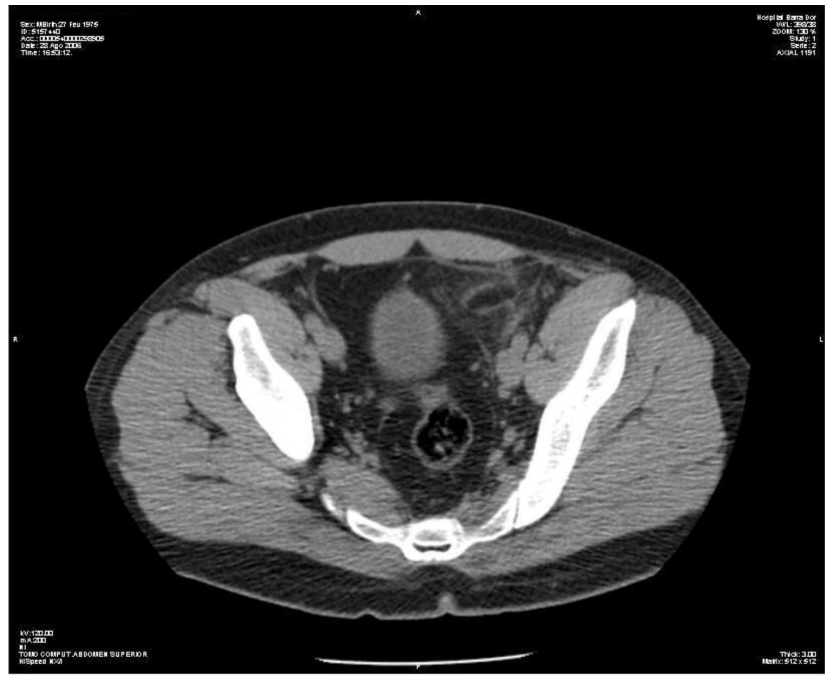

Figura 1 - TC abdome com imagem ovalar com densidade de gordura e centro radioluscente em cólon sigmóide.

Trabalho realizado no Hospital Barra D'Or - Rio de Janeiro - RJ - Brasil.

Recebido em 09/01/2008

Aceito para publicação em 14/02/2008 


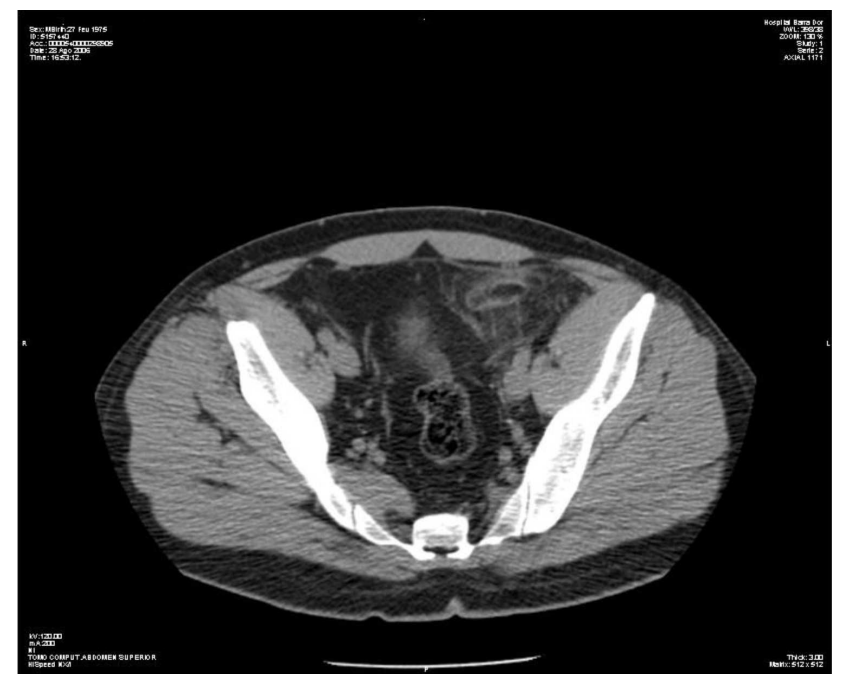

Figura 2 - TC abdome com imagem ovalar com densidade de gordura e centro radioluscente em cólon sigmóide, infiltração da gordura adjacente.

\section{DISCUSSÃO}

Os apêndices omentais são projeções de gordura na superfície externa do cólon que se projetam na cavidade peritoneal. Eles se encontram presentes em todos os segmentos colônicos, porém em maior densidade no cólon esquerdo. A apendagite epiplóica é uma condição clínica benigna que resulta da torção ou da trombose venosa espontânea das veias que drenam os apêndices. ${ }^{1}$ Atinge, principalmente, indivíduos entre a segunda e quinta décadas de vida, sem predomínio quanto ao sexo. ${ }^{1}$ A apendagite foi reconhecida como entidade nosológica em 1956 por Linn. ${ }^{2}$

O quadro clínico habitual é a presença de dor abdominal aguda localizada em quadrante inferior esquerdo, em paciente com bom estado geral e afebril.

O laboratório se caracteriza pela contagem de leucócitos e VHS normais ou pouco elevados. Atualmente, o diagnóstico é feito por tomografia computadorizada (TC). O achado tomográfico de imagem paracólica, ovalar, variando de 1 a $5 \mathrm{~cm}$, com densidade de gordura, associado a espessamento do revestimento peritoneal, e a atenuação da gordura periapendicular define o diagnóstico. ${ }^{3,4}$ Anteriormente ao advento da TC, o diagnóstico era intraoperatório. Há relatos de diagnóstico por ultra-sonografia (US) e ressonância nuclear magnética.

As possibilidades de diagnósticos diferenciais são variadas e incluem apendicite, diverticulite, ruptura de cisto ovariano, torção de ovário, gravidez ectópica, câncer de cólon, abcesso, ileíte de Crohn e adenite mesentérica. O quadro clínico pode mimetizar quadro de abdome agudo. ${ }^{5}$

O tratamento é conservador e ambulatorial. Consiste na administração de analgésicos e antiinflamatórios, com a melhora completa dos sintomas em torno de 3 a 14 dias. ${ }^{6} \mathrm{O}$ diagnóstico incorreto pode levar a intervenções desnecessárias, sejam elas hospitalizações, antibioticoterapia ou até mesmo cirurgias. A cirurgia só estaria justificada como forma de prevenir a recorrência e aderências adjacentes ao local de múltiplas inflamações, ou quando não há possibilidade de confirmação diagnóstica pela indisponibilidade de recursos diagnósticos avançados. A via preferencial seria a videolaparoscópica, com ligadura e excisão do apêndice inflamado. As vantagens do tratamento minimamente invasivo seriam a rápida recuperação após a intervenção, no entanto mesmo a via laparoscópica pode estar associada a graves complicações, tais como: sangramento excessivo, infecção, perfuração acidental de vísceras ocas ou reações adversas à anestesia geral. ${ }^{7}$ Portanto, a possibilidade destas complicações não encoraja o tratamento cirúrgico para esta condição de evolução benigna.

ABSTRACT: Appendagitis is an uncommon inflammatory abdominal disease, with good prognosis; nowadays it is being diagnosed more frequently because of advances in imaging methods. Clinical finding most often described was abdominal pain over left lower quadrant. Diagnosis is obtained through computed tomography. Recovery is uneventful under conservative treatment.

Key words: Abdominal Pain; Diagnosis; Epiploic Appendagitis; Treatment.

\section{REFERÊNCIAS}

1. Legome EL, Sims C, Rao PM. Epiploic appendagitis: adding to the differential of acute abdominal pain. J Emerg Med 1999; 17: 823-6.
2. Melo ASA, Moreira LBM, Pinheiro RA. Apendicite epiplóica: aspectos na ultra-sonografia e na tomografia computadorizada. Radiologia Brasileira 2002; vol.35(3): 171-174. 
3. Kantarci M, Duran C, Sirvanci M. Images of interest. Gastrointestinal: epiploic appendagitis. J Gastroenterol Hepatol 2005; 20 (3): 482.

4. Varela U, Fuentes MV, Rivadeneira R. Procesos inflamatorios del tejido adiposo intraabdominal, causa no quirurgica de dolor abdominal agudo: hallazgos en tomografia computada. Rev. Chil. Radiol 2004;10:28-34.

5. Singh AK, Gervais D, Hahn PF, Sagar P, Mueller PR, Novelline R. Acute epiploic appendagitis and its mimics. Radiographics 2005; 25(6):1521-34.

6. Vinson DR. Epiploic appendagitis: a new diagnosis for the emergency physician. Two cases report and a review. J Emerg Med 1999;17:827-32.
7. Sand M, Gelos M, Bechara FG, Sand D, Wiese TH, Steinstraesser L, Mann B. Epiploic appendagitis—clinical characteristics of an uncommon surgical diagnosis. BMC Surg. 2007; 1:7-11.

\section{Endereço para correspondência:}

Av. Ayrton Senna

Barra da Tijuca - Rio de Janeiro - Brazil

CEP:22775-001

Tel.: (21) 2430-3600

E-mail: guspignaton@gmail.com 